\title{
nate \\ Quantum Fisher Information of W and GHZ State Superposition under Decoherence
}

\author{
Volkan Erol \\ Okan University Computer Engineering Department Istanbul, Turkey; volkan.erol@gmail.com
}

\begin{abstract}
We study the changes in quantum Fisher information (QFI) values for one quantum system consisting of a superposition of W and GHZ states. In a recent work [6], QFI values of this mentioned system studied. In this work, we extend this problem for the changes of QFI values in some noisy channels. We show the change in QFI depending on noise parameters. We report interesting results for different type of decoherence channels.
\end{abstract}

Keywords: quantum fisher information; W state; GHZ state; decoherence

\section{Introduction}

The Quantum Information Theory and Quantum Computation are hot working areas that are the theoretical basis of Quantum Computers, which are described as computer technology of the future and intended to operate at very high speeds.

Quantum Fisher Information, a version of Fisher Information, developed for quantum systems, has also become a highly studied subject in recent years, as it also measures the sensitivity that systems can provide for phase sensitive tasks [1-35].

Quantum Fisher Information (QFI) is a very useful concept for analyzing situations that require phase sensitivity. This feature has attracted attention and extends the classical Fisher Information. Especially for systems with a higher QFI value, the accuracy is more clearly achieved; For example, clock synchronization [40] and quantum frequency standards [41]. Although some of the pure entangled systems may exceed the classical limit, this does not apply to all entangled systems [36]. The interaction between the quantum system and the environment not only reduces entanglement but also reduces the system's Quantum Fisher Information, in general. So we can say that researching quantum systems on QFI is important for the progress of quantum technologies. In recent studies, a single parameter, $\chi^{2}$ parameter, phase sensitivity was added to measure only the self-knowledge of the system under investigation [11]. Since a condition of $\chi^{2}<1$ is not provided for a general quantum system, it is understood that the system has multiple entanglement and this system provides better phase accuracy than a separable system. These quantum systems are called "useful" systems in the literature. For two-level N-particle quantumsystems, the Cramer-Rao limit is defined by the following formula [37,38]:

$$
\Delta \phi_{Q C B} \equiv \frac{1}{\sqrt{N_{m} F}}
$$

where $N_{m}$ is the number of experiments on the system being measured and $F$ is the Quantum Fisher Information value. We can write 3-dimensional vectors normalized in the nth direction of angular momentum operators, $J_{n}$, Pauli matrices as follows:

$$
J_{\vec{n}}=\sum_{\alpha=x, y, z} \frac{1}{2} n_{\alpha} \sigma_{\alpha}
$$


For $J_{n}$, the Fisher Information of the @ quantum system can be expressed in a symmetric matrix C [10]:

$$
F\left(\rho, J_{\vec{n}}\right)=\sum_{i \neq j} \frac{2\left(p_{i}-p_{j}\right)^{2}}{p_{i}+p_{j}}\left|\left\langle i\left|J_{\vec{n}}\right| j\right\rangle\right|^{2}=\vec{n} C \vec{n}^{T}
$$

where $p_{i}$ and $|i\rangle$ represent the eigenvalues and eigenvectors of the $\rho$ system, respectively, and the matrix $C$ is defined as

$$
C_{k l}=\sum_{i \neq j} \frac{\left(p_{i}-p_{j}\right)^{2}}{p_{i}+p_{j}}\left[\left\langle i\left|J_{k}\right| j\right\rangle\left\langle j\left|J_{i}\right| i\right\rangle+\left\langle i\left|J_{i}\right| j\right\rangle\left\langle j\left|J_{k}\right| i\right\rangle\right]
$$

The largest $F$ value between the $N$ options is selected and averaged over $N$ particles. The Fisher Information value is calculated as the greatest eigenvalue of the $C$ matrix. This definition is expressed by the equation:

$$
\bar{F}_{\max }=\frac{1}{N} \max _{\bar{n}} F\left(\rho, J_{\bar{n}}\right)=\frac{\lambda_{\max }}{N} .
$$

\section{Studied Quantum System and Reported Results}

In this section, we introduce three qubit systems in decoherence channels. Particularly the system studied is superposition of a W and a GHZ state [6],

$$
\left|\varphi^{N}\right\rangle=\alpha\left|W^{N}\right\rangle+\sqrt{1-\alpha^{2}}\left|G H Z^{N}\right\rangle
$$

Here $N$ is the number of qubits, and $\alpha$ is the superposition coefficient. In this work, we consider the case for $N=3$. The decoherence channels are amplitude damping, phase damping and depolarizing respectively. Then we study the QFI of the three qubit system for different scenarios. As a first scenario, we take decoherence parameter $p$ as 0.1 and $\alpha$ as the variable of the system. In Figure 1, changes in QFI values for three channels are showed for $p=0.1$ constant value.

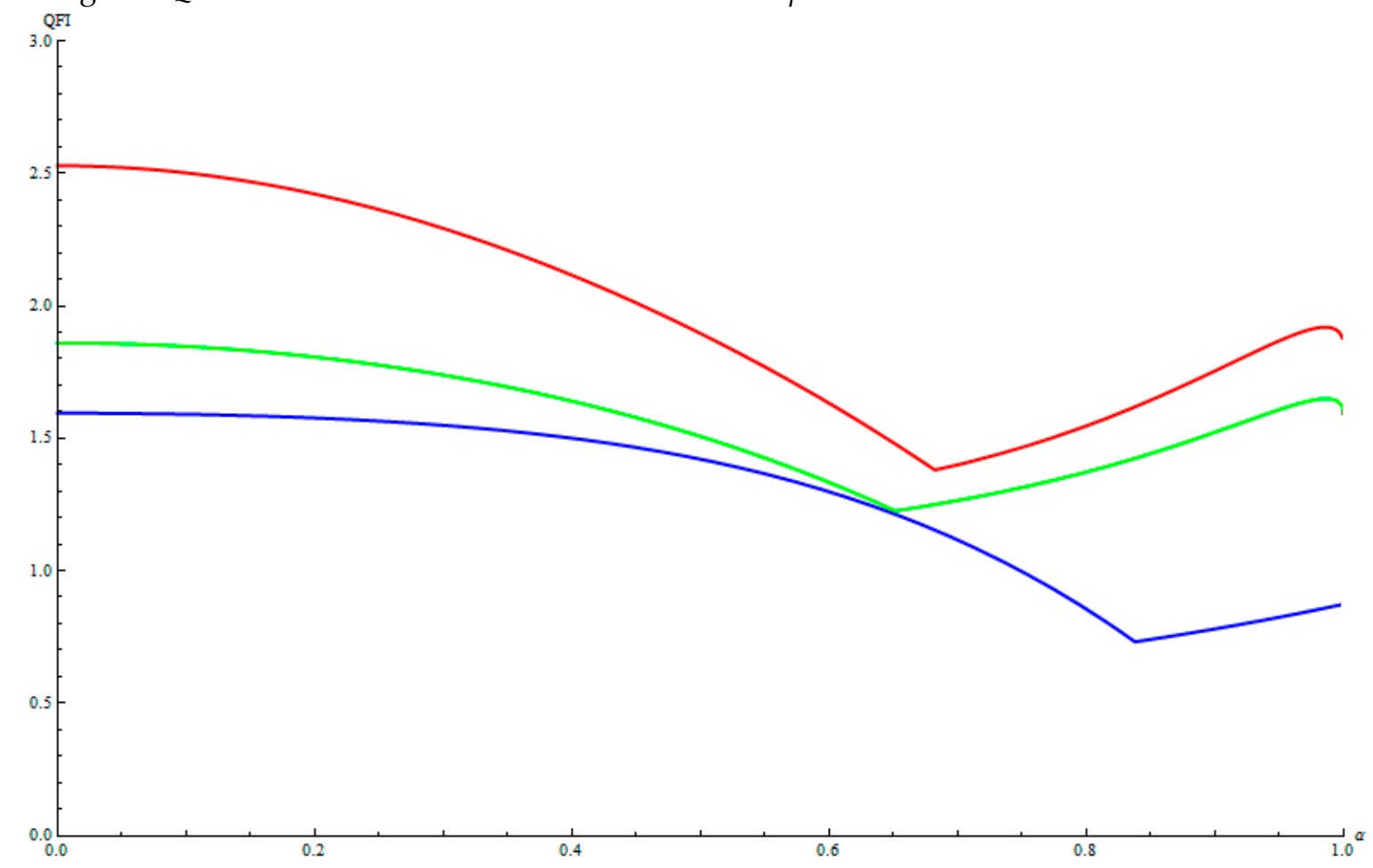

Fig. 1. Changes in QFI for decoherence channels for $p=0.1$ (Red: Amplitude Damping, Blue: Phase Damping, Green: Depolarizing) 
In the second scenario, we find the QFI as functions of $\alpha$ and $p$. We take values of $\alpha$ and $p$ between 0 and 1.

In Figure 2, we show in contour plot, the changes of QFI values in three channels.
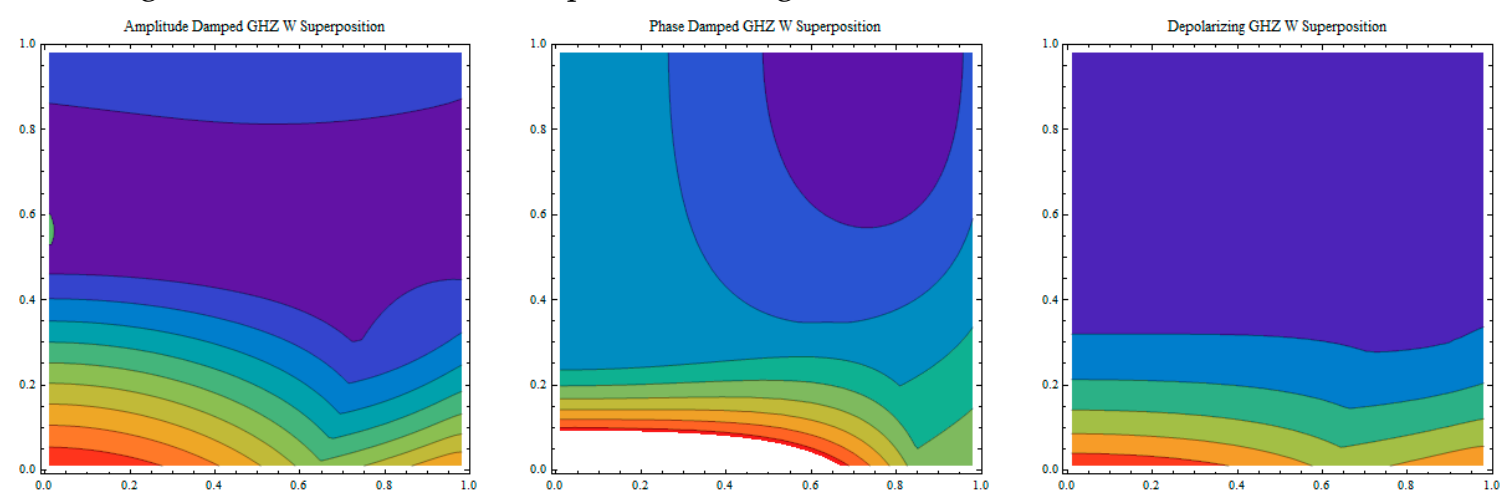

Fig. 2. QFI Values as a function of $\alpha$ and $\mathrm{p}$ (contour plot)

In Figure 3, we show in 3D Plots the changes in QFI values.
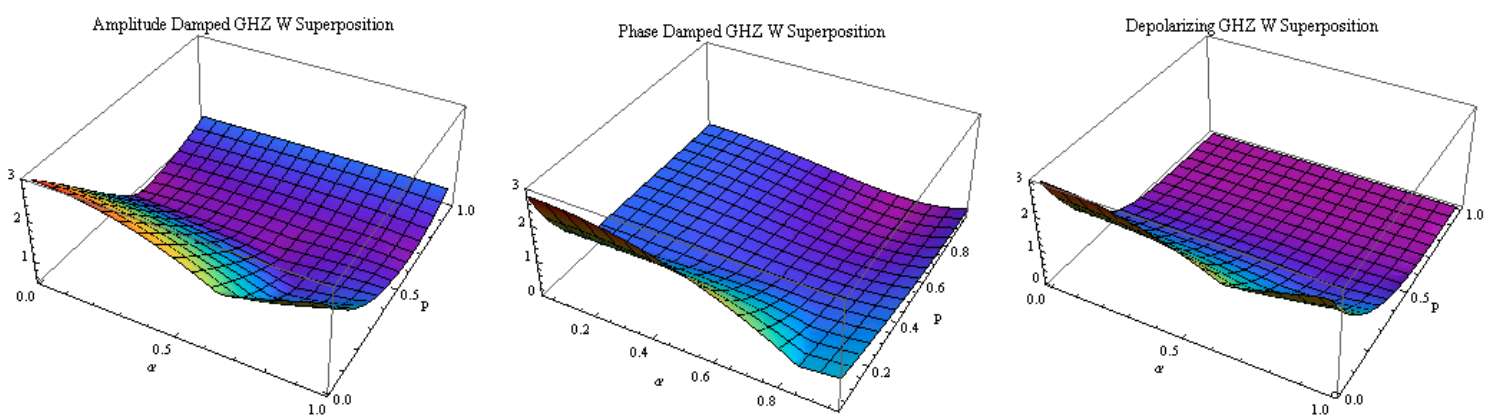

Fig. 3. QFI values as function of $\alpha$ and $\mathrm{p}$ (3D plot)

We have some observations about the results: firstly, regardless the value of the noise in the channel, dependence to $\alpha$ superposition coefficient effects the QFI values dramatically. In amplitude damping and depolarizing channel cases, it was observed that until to some values of $\alpha(\sim 0.65), \mathrm{QFI}$ value decreases and after this point, it starts increasing. In these cases, it behaves like a local minimum. This situation is not similar for phase damping case.

Second observation is that this superposition state is more resistant to phase damping channel. It can be viewed from Figures 2 and 3 (purple areas are smaller).

It was shown in [6] that this superposition is "useful" unless $\alpha$ is around 0.75 value.

\section{Conclusion}

We studied the changes in QFI values for a quantum system which is a superposition of $W_{3}$ and $\mathrm{GHZ}_{3}$ states. In this work, we extended this problem for the changes of QFI values in amplitude damping, phase damping and depolarizing channels. We showed the changes in QFI depending on noise parameters and superposition coefficient. We reported interesting results like the dramatical decreases of QFI values in amplitude damping and depolarizing channel cases. One of main observations arrived is that this nearly-useful state is more resistant to phase damping channel noise comparing to other decoherence channels.

\section{References}

1. Z. Ji et. al, Parameter Estimation of Quantum Channels, IEEE Trans. Info. Theory., vol. 54, no. 5172, 2008.

2. B. M. Escher, M. Filho, and L. Davidovich, General framework for estimating the ultimate precision limit in noisy quantum-enhanced metrology, Nat. Phys., vol. 7, no. 406, 2011.

3. X. Yi., G. Huang and J. Wang, Quantum Fisher Information of a 3-Qubit State, Int. J. Theor. Phys. vol. 51, p. $3458,2012$. 
4. N. Spagnolo et al., Quantum interferometry with three-dimensional geometry, Nature Sci. Rep., vol. 2, no. 862, 2010.

5. Z. Liu, Spin Squeezing in Superposition of Four-Kübit Symmetric State and W States, Int. J. Theor. Phys., vol. 52, p. 820, 2013.

6. F. Ozaydin, A. A. Altintas, S. Bugu and C. Yesilyurt, Quantum Fisher Information of N Particles in the Superposition of W and GHZ States, Int. J. Theor. Phys., vol. 52, p. 2977, 2013.

7. F. Ozaydin, A. A. Altintas, S. Bugu, C. Yesilyurt and M.Arik, Quantum Fisher Information of Several Qubits in the Superposition of A GHZ and two W States with Arbitrary Relative Phase, Int. J. Theor. Phys., vol. 53, p. 3259, 2014.

8. P. Gibilisco, D. Imparato, and T. Isola, Uncertainty principle and Quantum Fisher Information, J. Math. Phys., vol. 48, no. 072109, 2007.

9. A. Andai, Uncertainty principle with Quantum Fisher Information, J. Math. Phys., vol. 49, no. 012106, 2008.

10. J. Ma, Y. Huang, X. Wang and C. P. Sun, Quantum Fisher information of the Greenberger-Horne-Zeilinger state in decoherence channels, Phys. Rev. A, vol. 84, no. 022302, 2011.

11. Toth et al., Spin squeezing and entanglement, Phys. Rev. A, vol. 79, no. 042334, 2009.

12. F.Ozaydin, A.A.Altintas, S.Bugu, C.Yesilyurt, Behavior of Quantum Fisher Information of Bell Pairs under Decoherence Channels, Acta Phys. Pol. A, vol. 125(2), p. 606, 2014.

13. S.Z.Ang, G.I.Harris, W.P.Bowen and M.Tsang, Optomechanical parameter estimation", New J. Phys., vol. 15, no. 103028, 2013.

14. K.Iwasawa et al., Quantum-Limited Mirror-Motion Estimation, Phys. Rev. Lett., vol. 111, no. $163602,2013$.

15. M.Tsang, Quantum metrology with open dynamical systems, New J. Phys., no. 15, vol. 073005, 2013.

16. M.Tsang and N.Ranjith, Fundamental quantum limits to waveform detection, Phys. Rev. A, vol. 86, no. 042115, 2012.

17. M.Tsang, Ziv-Zakai Error Bounds for Quantum Parameter Estimation, Phys. Rev. Lett., vol. 108, no. 230401, 2012.

18. M.Tsang. H.M.Wiseman, C.M.Caves, Fundamental Quantum Limit to Waveform Estimation, IEEE Conference on Lasers and Electro-Optics (CLEO) 2011.

19. M.Tsang, J.H.Shapiro, S.Lloyd, Quantum Optical Temporal Phase Estimation by Homodyne Phase-Locked Loops, IEEE Conference on Lasers and Electro-Optics (CLEO) 2009.

20. F. Ozaydin, Phase damping destroys quantum Fisher information of W states, Phys. Let. A, vol. 378, pp. 3161-3164, 2014.

21. V. Erol, F. Ozaydin, A. A. Altintas, Analysis of entanglement measures and locc maximized quantum fisher information of general two qubit systems, Sci. Rep. 4, 5422, 2014.

22. F. Ozaydin, A. A. Altintas, C. Yesilyurt, S. Bugu, V. Erol, Quantum Fisher Information of Bipartitions of W States, Acta Physica Polonica A 127, 1233-1235, 2015.

23. V. Erol, S. Bugu, F. Ozaydin, A. A. Altintas, An analysis of concurrence entanglement measure and quantum fisher information of quantum communication networks of two-qubits, Proceedings of IEEE 22nd Signal Processing and Communications Applications Conference (SIU2014), pp. 317-320, 2014.

24. V. Erol, A comparative study of concurrence and negativity of general three-level quantum systems of two particles, AIP Conf. Proc. 1653 (020037), 2015.

25. V. Erol, F. Ozaydin, A. A. Altintas, Analysis of Negativity and Relative Entropy of Entanglement measures for qubit-qutrit Quantum Communication systems, Proceedings of IEEE 23rd Signal Processing and Communications Applications Conference (SIU2015), pp. 116-119, 2014.

26. V. Erol, Detecting Violation of Bell Inequalities using LOCC Maximized Quantum Fisher Information and Entanglement Measures, Preprints 2017, 2017030223 (doi: 10.20944/preprints201703.0223.v1), 2017.

27. V. Erol, Analysis of Negativity and Relative Entropy of Entanglement Measures for Two Qutrit Quantum Communication Systems, Preprints 2017, 2017030217 (doi: 10.20944/preprints201703.0217.v1), 2017.

28. V. Erol, The relation between majorization theory and quantum information from entanglement monotones perspective, AIP Conf. Proc. 1727 (020007), 2016.

29. V. Erol, A Proposal for Quantum Fisher Information Optimization and its Relation with Entanglement Measures, PhD Thesis, Okan University, Institute of Science, 2015.

30. F. Ozaydin, A. A. Altintas, Quantum Metrology: Surpassing the shot-noise limit with DzyaloshinskiiMoriya interaction, Sci. Rep. 5, 16360, 2015.

31. A. A. Altintas, Quantum Fisher Information of an open and noisy system in the steady state, Annals of Physics, 362, pp: 192-198, 2016.

32. F. Ozaydin, Quantum Fisher Information of a $3 \times 3$ Bound Entangled State and its Relation with Geometric Discord, International Journal of Theoretical Physics 54 (9), 3304-3310, 2015.

33. B. R. Frieden and R. A. Gatenby, Exploratory Data Analysis Using Fisher Information, Arizona, USA:Springer, 2007. 
34. L. Pezze, A. Smerzi, Entanglement, Nonlinear Dynamics, and the Heisenberg Limit, Phys. Rev. Lett., vol. 102, no. 100401, 2009.

35. V. Giovannetti, S. Lloyd and L. Maccone, Quantum-Enhanced Measurements: Beating the Standard Quantum Limit, Science, vol. 306, no. 1330, 2004.

36. P. Hyllus, O. Gühne and A. Smerzi, Not all pure entangled states are useful for sub-shot-noise interferometry, Phys. Rev. A, vol. 82, no. 012337, 2010.

37. C. W. Helstrom, Quantum Detection and Estimation Theory, New York: Academic Press, 1976.

38. A. S. Holevo, Probabilistic and Statistical Aspects of Quantum Theory, Amsterdam, The Netherlands: North-Holland, 1982.

39. H. N. Xiong, J. Ma, W. F. Liu and X. Wang, Quantum Fisher Information for Superpositions of Spin States, Quant. Inf. Comp., vol.10 no.5\&6, 2010.

40. R. Jozsa, D. S. Abrams, J. P. Dowling, and C. P. Williams, Quantum Clock Synchronization Based on Shared Prior Entanglement, Phys. Rev. Lett., vol. 85, no. 2010, 2000.

41. J.J.Bollinger, W. M. Itano, D. J. Wineland, and D. J. Heinzen, Optimal frequency measurements with maximally correlated states, Phys. Rev. A, vol. 54, no.R 4649, 1996. 